\title{
Extension of HCDstruct for Transonic Aeroservoelastic Analysis of Unconventional Aircraft Concepts
}

\author{
Jesse R. Quinlan* and Frank H. Gern ${ }^{\dagger}$ \\ NASA Langley Research Center, Hampton, VA, 23681
}

\begin{abstract}
A substantial effort has been made to implement an enhanced aerodynamic modeling capability in the Higher-fidelity Conceptual Design and structural optimization tool. This additional capability is needed for a rapid, physics-based method of modeling advanced aircraft concepts at risk of structural failure due to dynamic aeroelastic instabilities. To adequately predict these instabilities, in particular for transonic applications, a generalized aerodynamic matching algorithm was implemented to correct the doublet-lattice model available in Nastran using solution data from a priori computational fluid dynamics analysis. This new capability is demonstrated for two tube-and-wing aircraft configurations, including a Boeing 737-200 for implementation validation and the NASA D8 as a first use case. Results validate the current implementation of the aerodynamic matching utility and demonstrate the importance of using such a method for aircraft configurations featuring fuselage-wing aerodynamic interaction.
\end{abstract}

\section{Nomenclature}

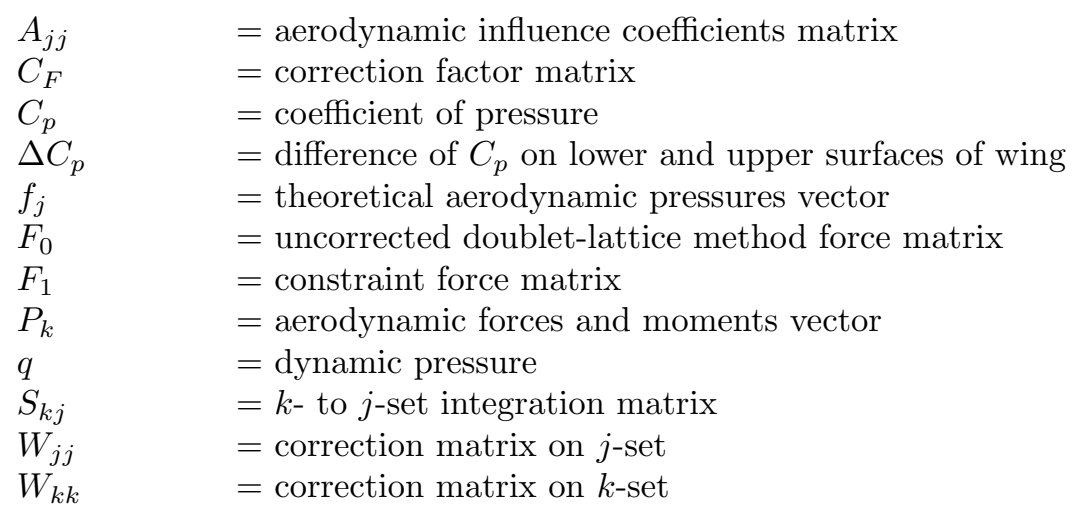

$\begin{array}{ll}\text { AATT } & =\text { Advanced Air Transport Technology } \\ \text { AESURF } & =\text { Nastran aerodynamic control surface } \\ \text { AIC } & =\text { aerodynamic influence coefficients } \\ \text { AOA } & =\text { angle of attack } \\ \text { BLI } & =\text { boundary layer ingestion } \\ \text { BWB } & =\text { blended wing body } \\ \text { CAERO1 } & =\text { Nastran doublet-lattice method panel element } \\ \text { CAERO2 } & =\text { Nastran slender body element } \\ \text { CFD } & =\text { computational fluid dynamics } \\ \text { CFL } & =\text { Courant-Friedrichs-Lewy number } \\ \text { CONM2 } & =\text { Nastran concentrated mass element } \\ \text { CQUAD4 } & =\text { Nastran panel structural element }\end{array}$

*Aerospace Engineer, Aeronautics Systems Analysis Branch, 1 N Dryden Street, and AIAA Member.

${ }^{\dagger}$ Assistant Branch Head, Vehicle Analysis Branch, 1 N Dryden Street, and AIAA Member. 


$\begin{array}{ll}\text { DLM } & =\text { doublet lattice method } \\ \text { DMAP } & =\text { Nastran direct matrix abstraction program } \\ \text { DMI } & =\text { Nastran direct matrix input } \\ \text { ECFT } & =\text { Enhanced Correction Factor Technique } \\ \text { FEM } & =\text { finite element model } \\ \text { FLOPS } & =\text { Flight Optimization System } \\ \text { HCDstruct } & =\text { Higher-fidelity Conceptual Design and structural optimization } \\ \text { HWB } & =\text { hybrid wing body } \\ \text { LaRC } & =\text { Langley Research Center } \\ \text { MIT } & =\text { Massachusetts Institute of Technology } \\ \text { NASA } & =\text { National Aeronautics and Space Administration } \\ \text { ND8 } & =\text { NASA D8 } \\ \text { OML } & =\text { outer mold line } \\ \text { OpenVSP } & =\text { Open Vehicle Sketch Pad } \\ \text { PSHELL } & =\text { Nastran shell properties } \\ \text { RBAR } & =\text { Nastran rigid bar element } \\ \text { SA\&I } & =\text { Systems Analysis and Integration } \\ \text { TW } & =\text { tube and wing }\end{array}$

\section{Introduction}

SIZING conventional aircraft concepts is commonly performed using low-order regression methods with $S$ databases of relevant configuration data, such as those used in the Flight Optimization Performance System (FLOPS) tool. ${ }^{1}$ However, these methods are ill-suited for situations where the target concept is unconventional in one or more ways. For example, hybrid wing body (HWB) or blended wing body (BWB) aircraft concepts are fundamentally unconventional aircraft due to their highly-integrated structures and streamlined outer mold line (OML). Similarly, recent efforts to structurally size advanced tube and wing (TW) concepts, such as double-bubble fuselage configurations, ${ }^{2-4}$ as depicted in Fig. 1, using conventional sizing methods have suggested a need for more advanced approaches. Hence, the Higher-fidelity Conceptual Design and structural optimization (HCDstruct) tool ${ }^{2}$ was significantly enhanced to include a generalized TW airframe sizing capability, as described in Ref. 5. The current paper highlights the further development of methods required to realize dynamic aeroservoelastic analysis capabilities for these advanced aircraft configurations.

In order to properly predict flutter for advanced conceptual aircraft designs using HCDstruct, in particular for high Mach number simulations, a major enhancement to the aerodynamic modeling was necessary, whose implementation is described in Section II. Discussions of the aerodynamic matching algorithm and computational fluid dynamics (CFD) solver are included in Sections II.A and II.B, respectively. The use of this additional capability is demonstrated using the Boeing 737-200 and NASA double-bubble (ND8) configurations, where static aeroelastic analysis using an aerody-

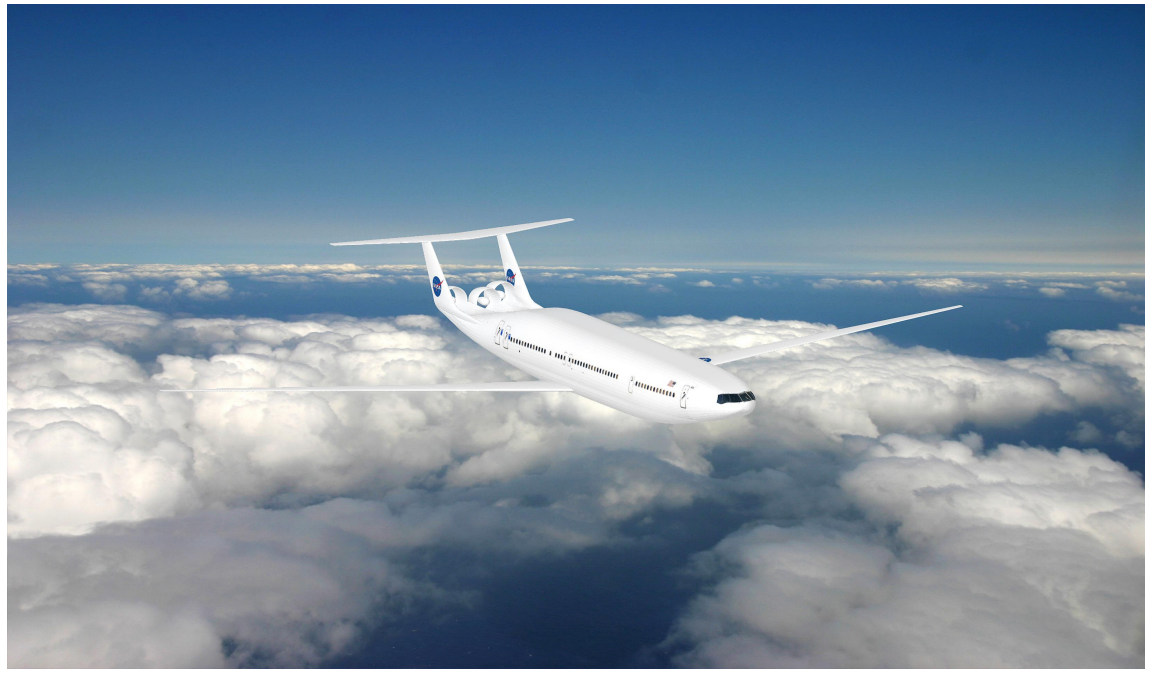

Figure 1. MIT's D8 aircraft concept featuring a double-bubble fuselage design (NASA Photo). 
namic correction based on corresponding CFD simulations is presented for each in Sections III and IV, respectively. A summary of this work is presented in Section V.

\section{HCDstruct Development}

HCDstruct was developed at NASA Langley Research Center (LaRC) to fill a critical analysis gap between high level, lower order approaches commonly used for conceptual design and the low level, detailed, often finite-element-based, optimization approaches commonly used for advanced preliminary design. Specifically, HCDstruct was developed to complement the FLOPS tool, which is a versatile, multidisciplinary computer program for conceptual and preliminary design and analysis of advanced aircraft concepts that relies on regression-based structural sizing algorithms. ${ }^{1,6}$ While the detailed finite-element-based sizing analyses often performed later in the design cycle, such as those for the HWB by Boeing, ${ }^{7}$ may theoretically offer additional insights, the computational resources required for these efforts often limit them to single-point design analysis. Thus, HCDstruct was developed to bridge the gap between the FLOPS regression-based sizing techniques and current state-of-the-art finite-element-based approaches to advanced preliminary design data. In fact, the tool has evolved to provide a means of optimizing the primary structure for the fuselage and wing for a given aircraft configuration using finite element methods while only requiring FLOPS-level user data.

Since an overview of the static aeroelastic analysis features of HCDstruct 2.0.0 may be found in Ref. 5, only those developments made in support of dynamic aeroservoelastic analysis for HCDstruct 2.1.0 are described in the current paper. Specifically, the theoretical development and implementation of the Enhanced Correction Factor Technique (ECFT) ${ }^{8,9}$ is described below in Section II.A. With this aerodynamic matching utility, hereafter referred to as aeroMatch, the linear aerodynamics predicted by the Doublet-Lattice Model (DLM) within Nastran are corrected using a pre-multiplier matrix computed using a priori solution data from corresponding computational fluid dynamics (CFD) simulations. Only with this aerodynamic correction may the significant drop in the flutter boundary during transonic flight, commonly referred to as the transonic dip, be captured using the Nastran DLM model formulation.

\section{A. Aerodynamic Matching}

Nastran DLM aerodynamic loads are applied to the model by splining them to the different structural components. The use of the Nastran DLM method for aerodynamic loads prediction is a well-established process used by aircraft manufacturers for design, loads analyses, and aircraft certification. However, with the DLM being a lifting surface method based on potential theory, it is by nature unable to properly reproduce viscous, three-dimensional, or transonic flow effects such as shocks, flow separation, and complex flows around control surfaces and high-lift devices. As a result, early in the development of the Nastran DLM, researchers derived processes to correct DLM aerodynamics to match available CFD or experimental data using correction factors or pre-multipliers to the aerodynamic influence coefficients (AIC), or $\left[A_{j j}\right]$, matrix in Nastran. ${ }^{10}$

The current approach uses a refinement of the initial correction factor technique outlined in Ref. 10, which was developed by researchers at the Raytheon Aircraft Company. Their Enhanced Correction Factor Technique (ECFT) addresses some of the shortcomings of the initial method from Ref. 10 by expanding the correction methodology from a diagonal matrix of correction factors to a full correction factor matrix. ${ }^{11}$ The ECFT is based on a modal decomposition of the aerodynamic downwash by forming a base matrix, $[W]$, which is composed of harmonic aerodynamic downwash vectors as described in Ref. 11. A correction factor matrix is then calculated by replacing given aerodynamic pressure data from CFD or wind tunnel testing in the uncorrected DLM force matrix, $\left[F_{0}\right]$, thus yielding a constraint force matrix, $\left[F_{1}\right]$, where

$$
\left[F_{1}\right]=\left[C_{F}\right]\left[A_{j j}\right][W]=\left[C_{F}\right]\left[F_{0}\right]
$$

The correction factor matrix, $\left[C_{F}\right]$, can then be obtained from

$$
\left[C_{F}\right]=\left[F_{1}\right]\left[F_{0}\right]^{-1}
$$

The ECFT method derives the correction factor matrix, $\left[C_{F}\right]$, from pressure data, thus yielding an equivalent to the $\left[W_{j j}\right]$ correction matrix in the Nastran $j$-set (see Fig. 2). However, actual correction factors in Nastran 
are applied in the $k$-set by correcting the DLM box-wise lift force and moment distributions via the correction factor matrix $\left[W_{k k}\right]$, where the matrix sizes are given by $k=2 j .{ }^{11,12}$

Further improvements to the ECFT have been made at the Gulfstream Aerospace Corporation by deriving a diagonal ECFT which is diagonally dominant and therefore has a more physical meaning. ${ }^{9}$ The Gulfstream reference also outlines a path for computing the $\left[W_{k k}\right]$ matrix from $\left[W_{j j}\right]$. A slightly different procedure based on the assumptions in Ref. 9 is used here and is described below.

The Nastran DLM force and moment data are computed from the DLM box pressures via the Nastran integration matrix $\left[S_{k j}\right]:^{9,12}$

$$
P_{k}=\left[W_{k k}\right]\left[S_{k j}\right] f_{j}=q\left[W_{k k}\right]\left[S_{k j}\right]\left[A_{j j}\right]^{-1} w_{j}
$$

or

$$
P_{k}=\left[S_{k j}\right]\left[W_{j j}\right] f_{j}=q\left[S_{k j}\right]\left[W_{j j}\right]\left[A_{j j}\right]^{-1} w_{j}
$$

where $P_{k}$ is the vector of aerodynamic forces and moments, $\left[S_{k j}\right]$ is the integration matrix, $q$ is the dynamic pressure, and $f_{j}$ are the theoretical aerodynamic pressures. As outlined in Ref. 9, the relationship between $\left[W_{k k}\right]$ and $\left[W_{j j}\right]$ is then given by

$$
\left[W_{k k}\right]\left[S_{k j}\right]=\left[S_{k j}\right]\left[W_{j j}\right]
$$

which is an indeterminate system. The correction factor matrix, $\left[W_{k k}\right]$, (see Fig. 2) is then obtained by virtue of the procedure outlined in Ref. 9 and supplied to Nastran through a direct matrix input (DMI) WKK data card.

\section{B. CFD Simulation}

Development of aeroMatch was performed generally independent of the choice of CFD solver, as the intent was to make the utility extensible to a wide variety of applications. Hence, aeroMatch relies on a generic Tecplot ${ }^{13}$ formatted (.dat) triangulated surface solution file. For the current efforts, the Cart3D CFD solver ${ }^{14}$ was used to provide this surface solution file. Cart3D is an inviscid, unstructured, Cartesian-based CFD solver developed at NASA Ames Research Center and is used widely throughout the government and industry for conceptual and preliminary aerodynamic design and analysis. For the current paper, the mesh adaptation features of Cart3D were not used; rather, the refinement level was used to manually confirm mesh convergence.

The aircraft geometry was modeled using Open Vehicle Sketch Pad (OpenVSP), ${ }^{15}$ from which a hermite representation of the OML could be easily extracted. Details of the translation of the hermite representation of the OML to the triangulated surface mesh input to Cart3D can be found in Ref. 16. All Cart3D simulations for the current paper were performed on a local, shared-memory, Linux cluster of 40 nodes. Simulations were performed using a five-stage Runga Kutta scheme, with a Courant-Friedrichs-Lewy (CFL) number of 1.0 and van Leer flux limiter. A symmetry boundary condition was applied at the spanwise centerline, inflow conditions were specified for the freestream at the inflow plane, and far-field conditions were applied at

the remaining boundaries. The boundaries of the Cartesian box were specified at more than five times the corresponding dimensions of the OML.

\section{Boeing 737-200 Configuration}

The first application of the aeroMatch utility was the Boeing 737-200, as this represents a traditional, tube-and-wing aircraft with known flying characteristics and available validation data. A geometric model of the Boeing 737-200 was constructed using public sources and is described in Ref. 5. The same model of the 737-200, which is shown in Fig. 3, is used for the current study.

The primary objective of the current 737-200 analysis was to validate the implementation of the aeroMatch utility. Specifically, a $2.5 \mathrm{G}$ pull-up maneuver at a Mach number of 0.5 and dynamic pressure of 1.769 psi was selected for analysis with HCDstruct, as this load case is the primary wing sizing load case used in HCDstruct, and corresponding inviscid CFD simulations were performed using Cart3D. In Section III.A, the 737-200 aerostructural model constructed by HCDstruct is presented, and in Section III.B, the CFD analysis of the 737-200 is described. Finally, in Section III.C, the uncorrected results of the doublet-lattice method (DLM) aerodynamic model are compared to the corresponding CFD solution along with aerodynamically-corrected DLM data using aeroMatch. 


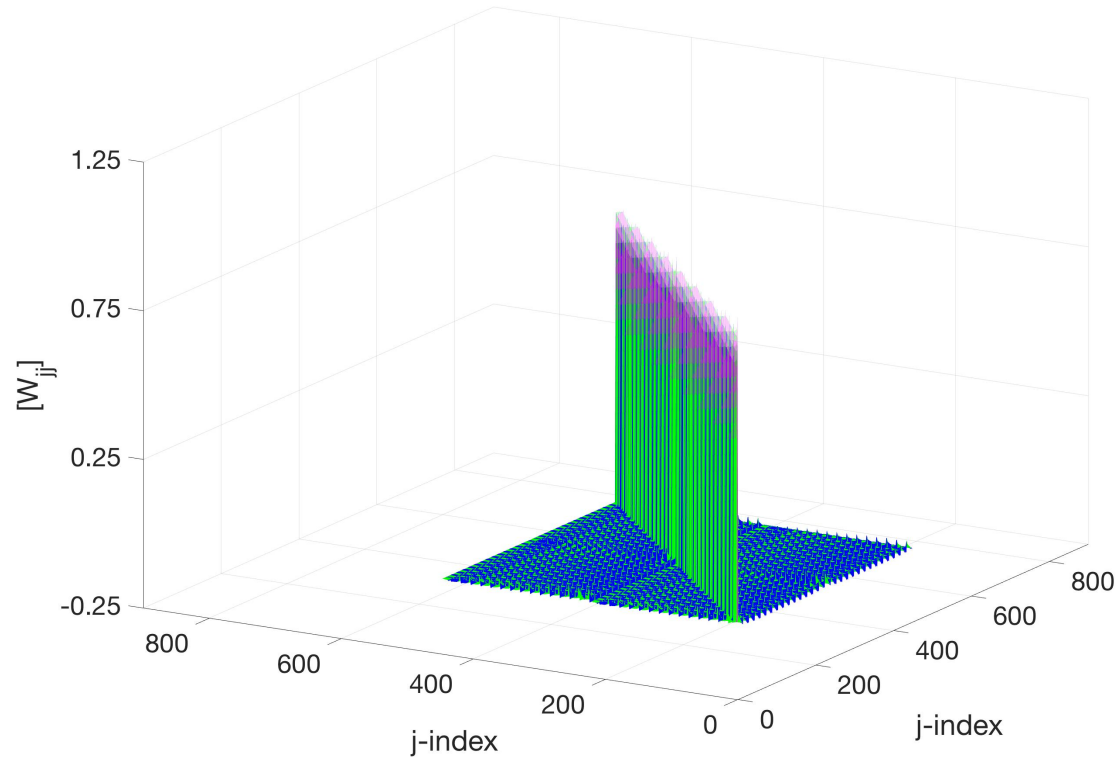

(a) $\left[W_{j j}\right]$ correction factor matrix.

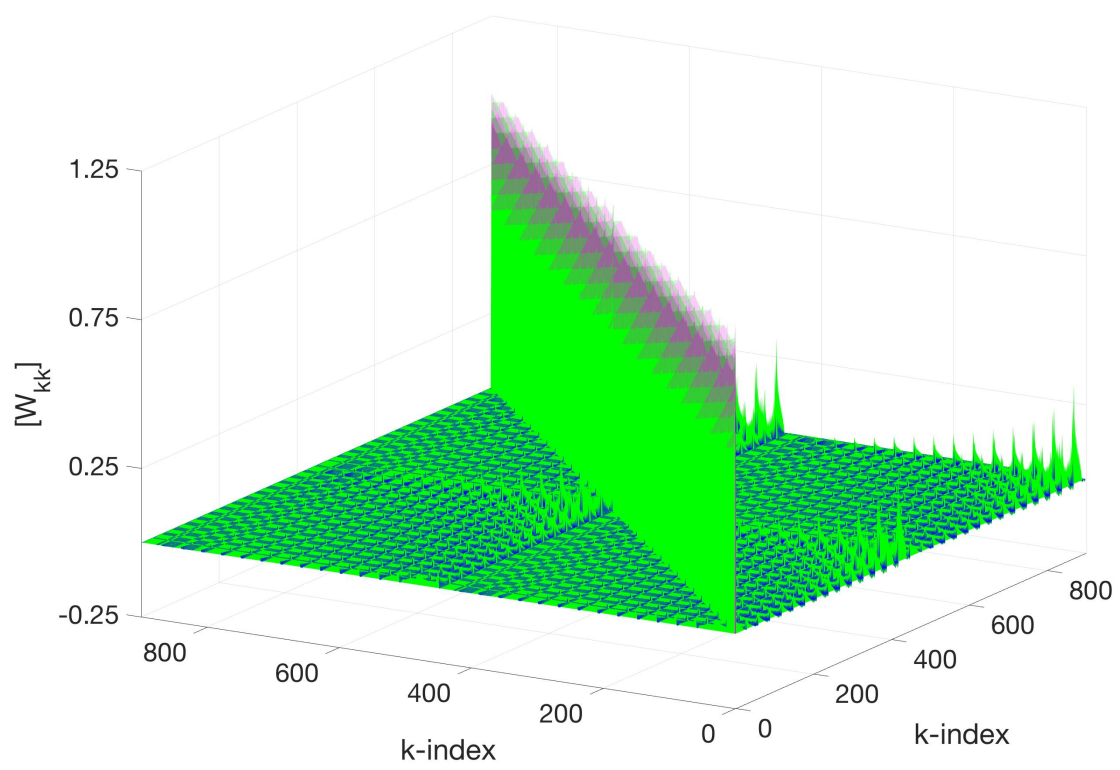

(b) $\left[W_{k k}\right]$ correction factor matrix.

Figure 2. Correction factor matrices for aerodynamic matching. Note that the size of $\left[W_{j j}\right]$ is $j \mathbf{x} j$, while the size of $\left[W_{k k}\right]$ is $k \times x k$, where $k=2 j$. 


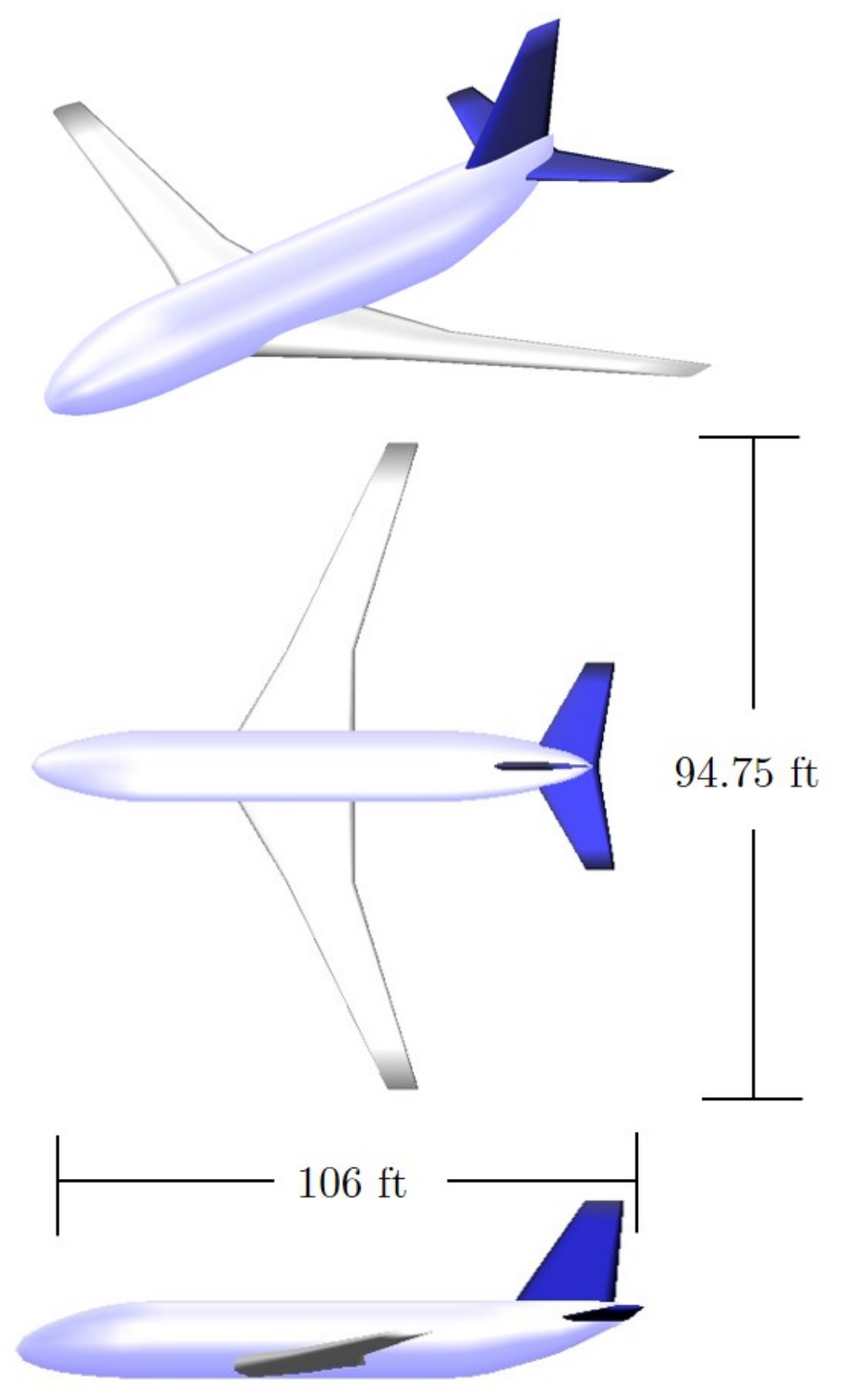

Figure 3. A three-view of the 737-200 model rendered using OpenVSP. 


\section{A. Aerostructural Model}

A three-dimensional aeroelastic model of the 737-200 is presented in Fig. 4, where the primary structural elements and aerodynamic model panels are rendered, along with the rigid bars and concentrated masses for modeling the empennage, propulsion system, and gear inertial loads. Additional details of this model can be found in Ref. 5. For the current study, the default HCDstruct SOL 200 analysis was used to optimize the structural weight via shell element thickness. Upon reaching a minimum structural weight, the optimized distribution of shell thicknesses were used to build a structurally-optimal aeroelastic model for targeted static aeroelastic analysis us-

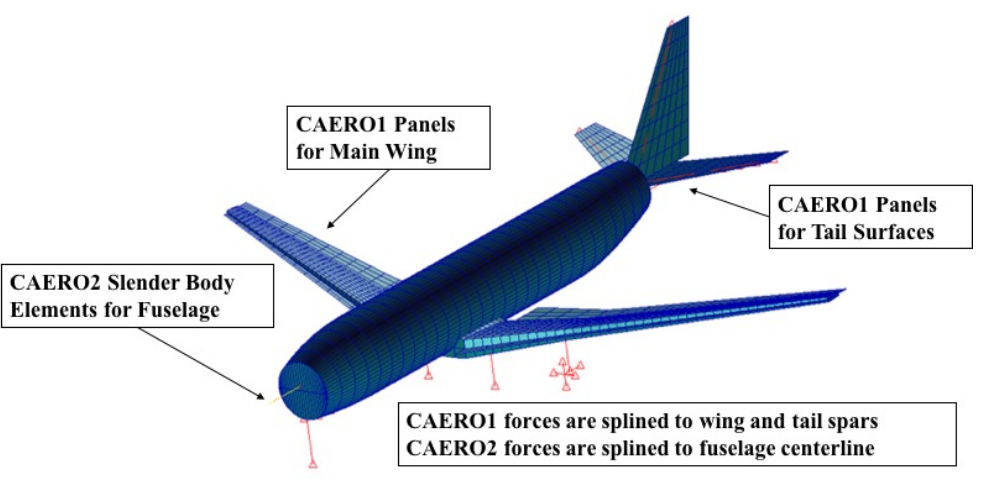

Figure 4. An annotated depiction of the 737 structural FEM showing relevant model features. Note that the slender body elements used to aerodynamically model the fuselage cannot be rendered by MSC Patran/FlightLoads. ing SOL 144. For this model, the angle of attack $(\mathrm{AOA})$ was fixed to match that of the trimmed 2.5G maneuver, and the aerodynamic output via MONCNCM was confirmed to match that output during the SOL 200 optimization. Since the purpose of this study is to demonstrate the ECFT implementation for aeroMatch, the model was rigidized to facilitate comparisons to the corresponding CFD analysis, presented in the following section. Hence, following the MONCNCM comparison, the model was rigidized by increasing the material stiffness of all structural elements by six orders of magnitude, at which point the computed rigid aerodynamic forces output by Nastran matched that of the elastic aerodynamic forces to within five significant figures. This structurally-optimized, rigidized aeroelastic model was used in conjunction with a custom direct matrix abstraction program (DMAP) routine and SOL 144 to calculate the corresponding AIC matrix, $\left[A_{j j}\right]$, needed for the ECFT method, and all subsequent results comparisons are made for this structurally-optimized, rigidized aeroelastic model. A summarized flow chart describing the current analysis approach is presented in Fig. 5.

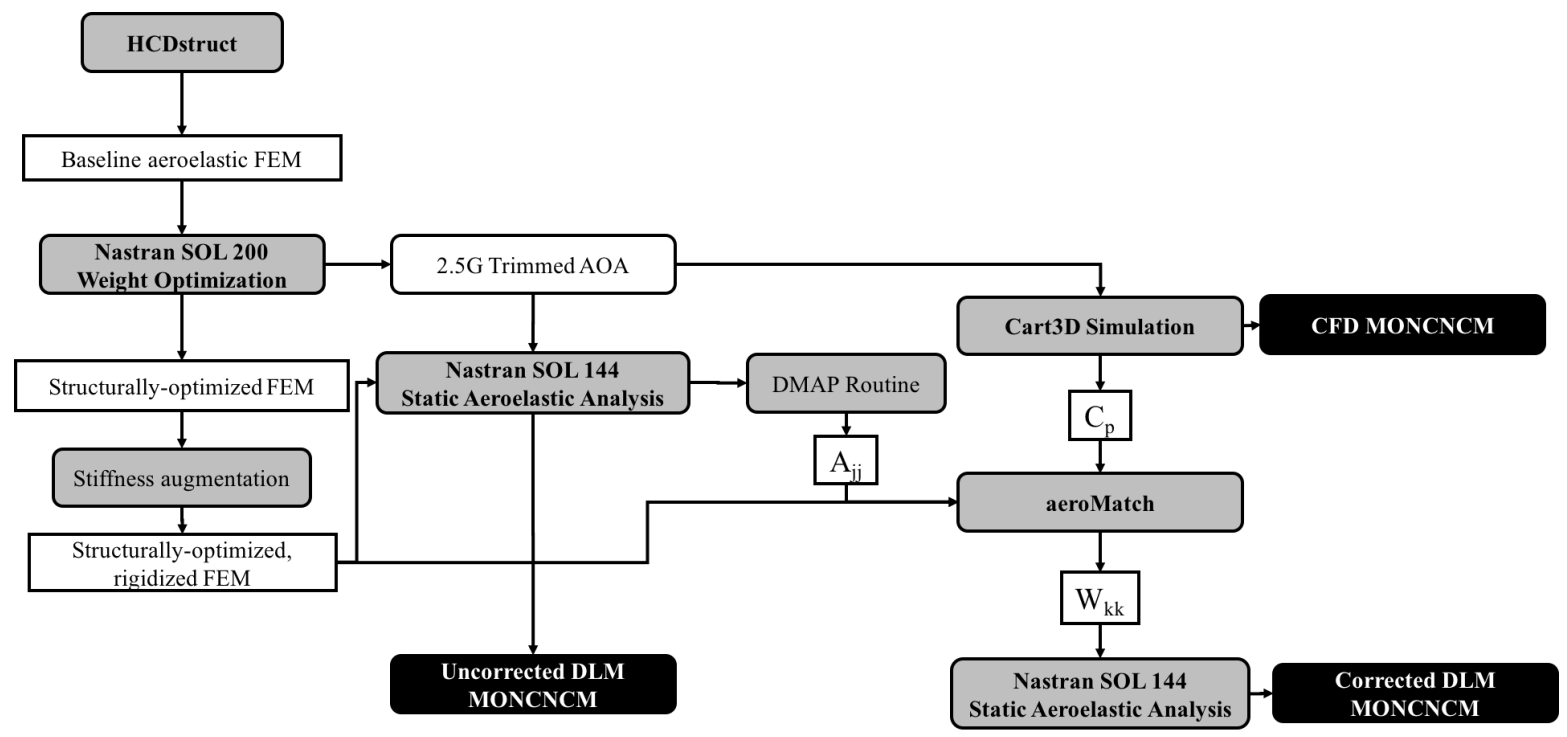

Figure 5. A flow chart summarizing the problem formulation used to validate the ECFT implementation in the aeroMatch utility. 


\section{B. CFD Analysis}

Three-dimensional, inviscid, steady-state CFD simulations of the 737-200 model shown in Fig. 3 were performed using Cart3D in order to build a truth surface pressure solution with which aeroMatch could be used to formulate a $\left[W_{k k}\right]$ correction matrix. With the corresponding coefficient of pressure, $C_{p}$, surface solution, the difference of the upper and lower wing surface $C_{p}$, or $\Delta C_{p}$, was computed at each DLM panel for the main wing using aeroMatch. Solver configuration details were discussed in Section II.B; in this section, details of the computational mesh and solution are presented.

Three levels of mesh refinement were used to confirm mesh convergence, corresponding to 13, 14, and 15 levels of refinement beyond the initial Cartesian mesh supplied to Cart3D. The finest mesh solution, corresponding to 15 refinements, is used from this point forward. A depiction of the spanwise centerplane mesh is shown in Fig. 6, along with the surface mesh colored by contours of $C_{p}$. The computational mesh included approximately 130.7 million cells. The surface mesh was tailored to provide smooth transitions between aircraft components, such as at the wing and fuselage intersection, and to cluster cells around leading edges of the lifting surfaces, as shown in Fig. 7.

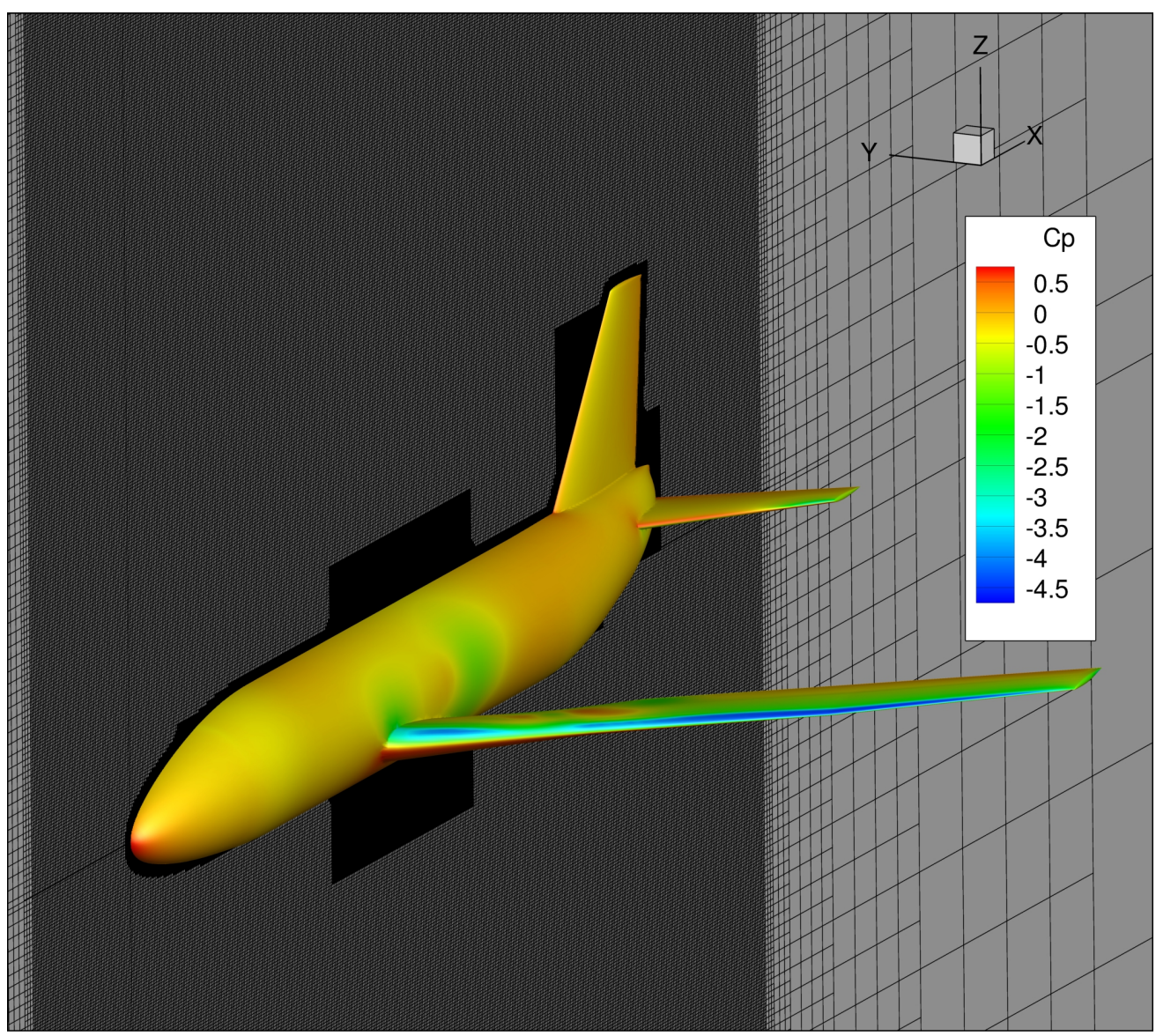

Figure 6. An illustration of the spanwise centerplane mesh around the surface of the 737-200, where the model surface is shown colored by contours of $C_{p}$.

The 737-200 was simulated at an AOA of $13.2^{\circ}$, which corresponded to the trimmed $2.5 \mathrm{G}$ maneuver for the structurally-optimized, rigid aeroelastic model described in the previous section. The Cart3D solver was executed until the residual and integrated forces remained unchanged, which required approximately 300 iterations at a CFL number of 1.0. The $C_{p}$ solution is shown in Fig. 8 for the entire vehicle. This $C_{p}$ surface solution was used by aeroMatch to build a corresponding $\left[W_{k k}\right]$ matrix, which is described in the next section. 


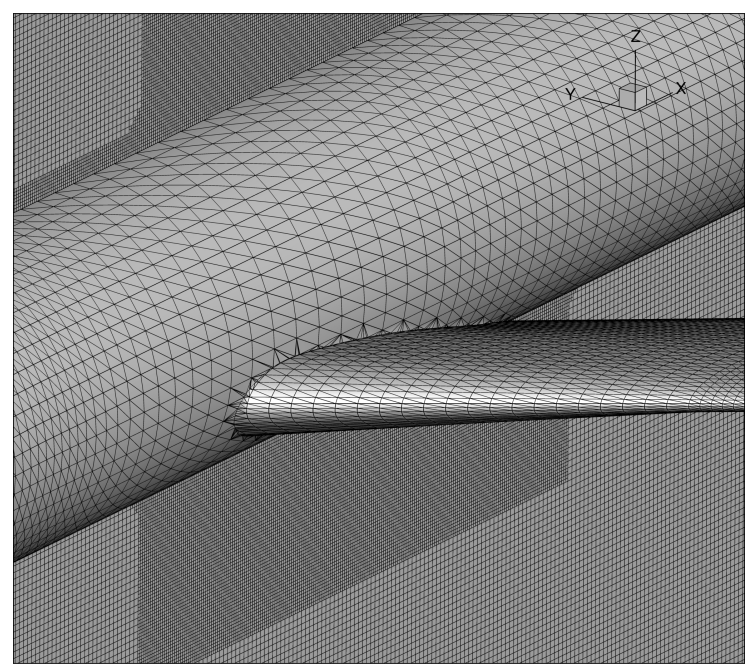

(a) Surface mesh detail at wing-fuselage intersection.

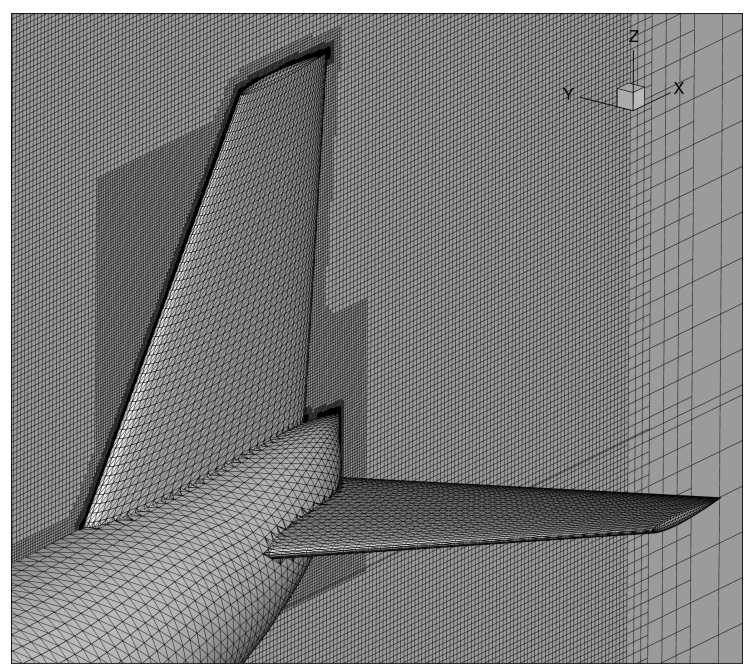

(b) Surface mesh detail at empennage.

Figure 7. Surface mesh illustrations showing the relative cell clustering at component intersections for the 737-200 model.

\section{Results}

In order to validate the implementation of the ECFT presented in Section II.A, aeroMatch was used in conjunction with the 737-200 $C_{p}$ CFD solution shown in Fig. 8 to construct [ $W_{k k}$ ], which is shown in Fig. 9. For the current implementation, the ECFT correction was only applied to the main wing; thus the resulting main wing sub-matrix of the $\left[W_{k k}\right]$ was augmented by an identity matrix such that the tail lifting surfaces and fuselage slender body elements were unaffected by the aerodynamic correction process, as can be seen by comparing the diagonal and off-diagonal elements of $\left[W_{k k}\right]$ in Fig. 9, noting that the main wing sub-matrix represents the first $900 \times 900$ elements of $\left[W_{k k}\right]$ for the 737-200 model.

Comparisons of the sectional lift coefficient for the main wing for the uncorrected DLM model (red line/squares), the Cart3D CFD solution (black line/diamonds), and the aeroMatch-corrected DLM (blue line/triangles) are shown in Fig. 10. Also shown in this figure is a streamwise perspective of the 737-200 model overlaid by contours of $C_{p}$ for referencing the spanwise data locations on the line plots of MONCNCM. The uncorrected DLM solution, although only plotted for the main wing DLM panels, includes the effects of fuselage-wing interaction from the slender body elements used to model the fuselage, which was accomplished by including the wing DLM panels in the fuselage interference group. While the outboard uncorrected DLM, CFD, and corrected DLM data appear to agree qualitatively near the wing tips, the inboard regions of the wing show considerable disagreement. Unlike the uncorrected DLM results, the CFD solution suggests considerable lift is generated by the fuselage and subsequently by the wing due to its interaction with the fuselage. At the midsection wing break, a considerable drop in sectional lift coefficient is shown in the CFD data that is not reflected in the uncorrected DLM data. Although this wide discrepancy was at first concerning, consulting Figs. 11 and 12 in Ref. 17 confirms that the CFD solution is consistent with wind tunnel and flight data reported for the 737-300 aircraft, whose OML is nearly identical to the 737-200. This observation further supports the inadequacy of using uncorrected DLM aerodynamic modeling for aircraft configurations where fuselage-wing interaction and three-dimensional flow are to be expected, which is clearly the case for the 737-200 at this flight condition.

For the purpose of validating the implementation of the aerodynamic matching capability in aeroMatch, the most important result to be gleaned from Fig. 10 is the comparison of the Cart3D CFD solution data and the corrected DLM data. Comparing these two curves demonstrates the efficacy of aeroMatch. Despite the considerable disagreement between the uncorrected DLM data and the Cart3D CFD solution, aeroMatch constructed a $\left[W_{k k}\right]$ correction matrix that when used with SOL 144 resulted in a sectional lift distribution over the main wing in good agreement with the CFD solution. For this study, the correction method was applied only to the pitch mode of the aerodynamic downwash decomposition matrix in the ECFT method; however, the same process can be used to build more general correction matrices. 

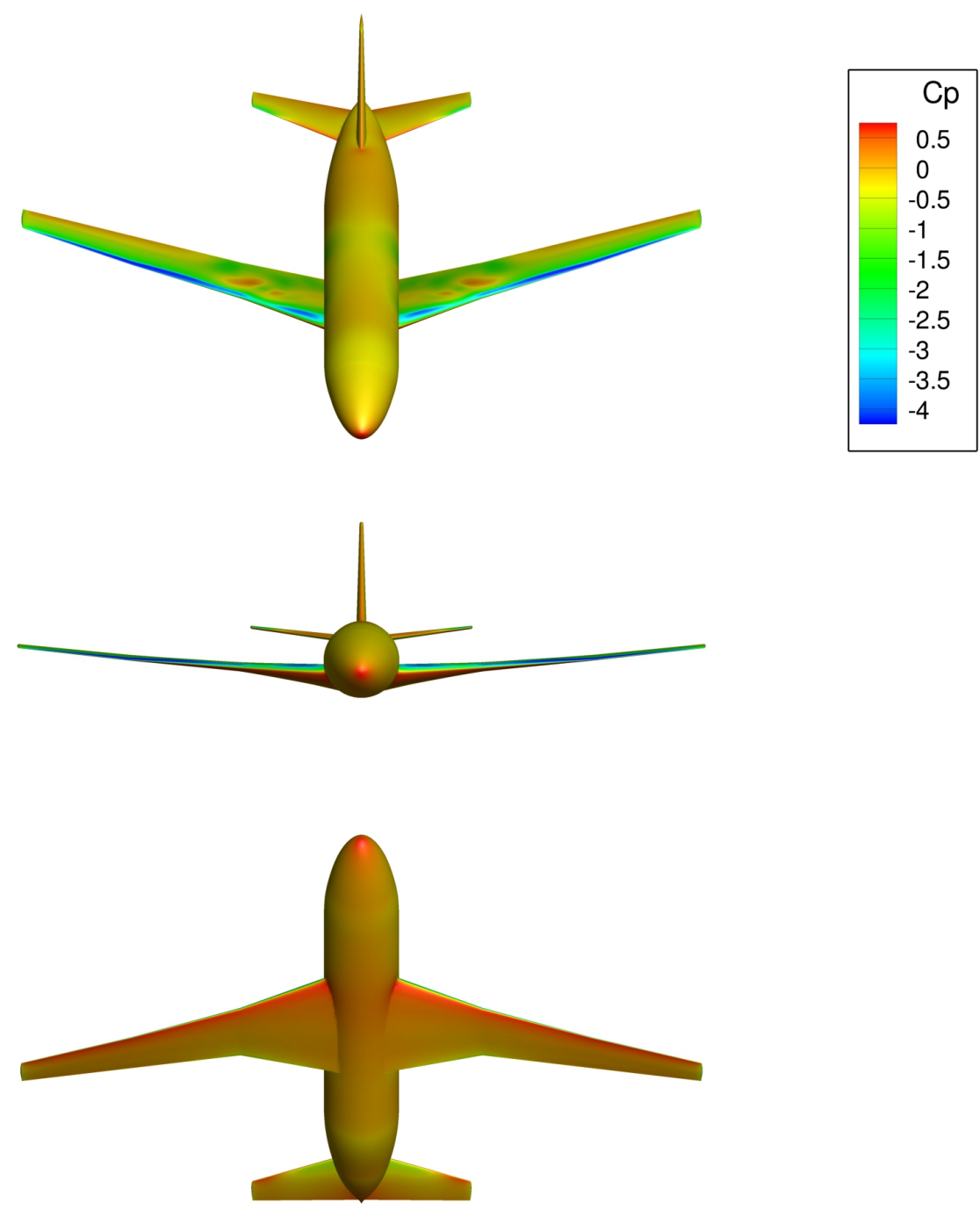

Figure 8. The 737-200 $C_{p}$ surface solution for the fine mesh computed using Cart3D at an AOA of $13.2^{\circ}$. 


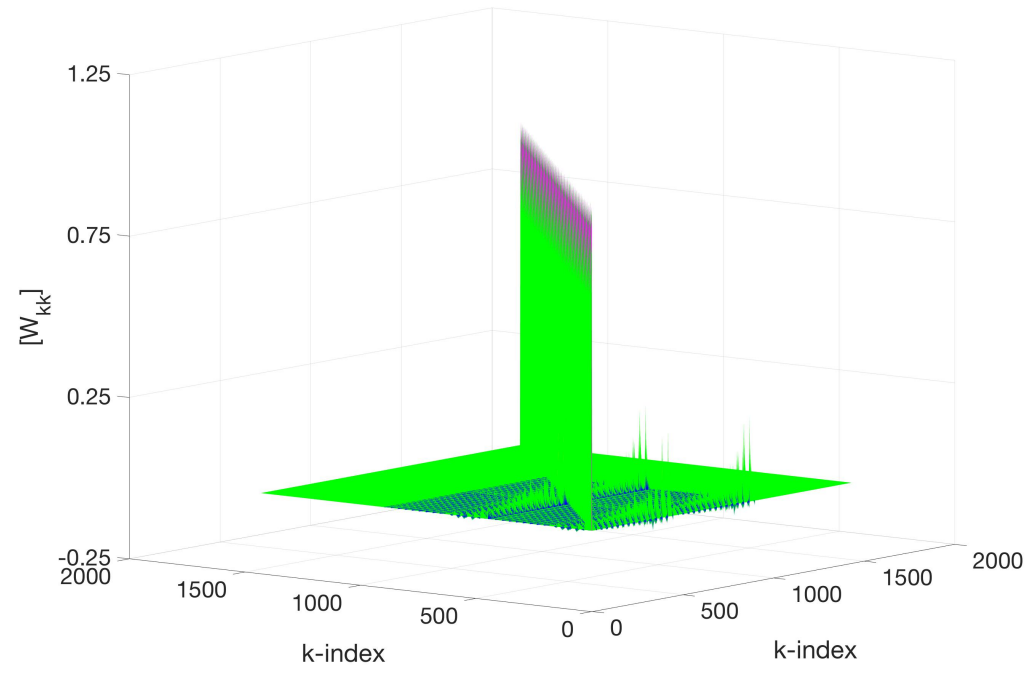

Figure 9. Surface plot of the $\left[W_{k k}\right]$ matrix as computed using aeroMatch for the 737-200 model. The horizontal axes represent the $k$-index, and the vertical axis represents $\left[W_{k k}\right]$.

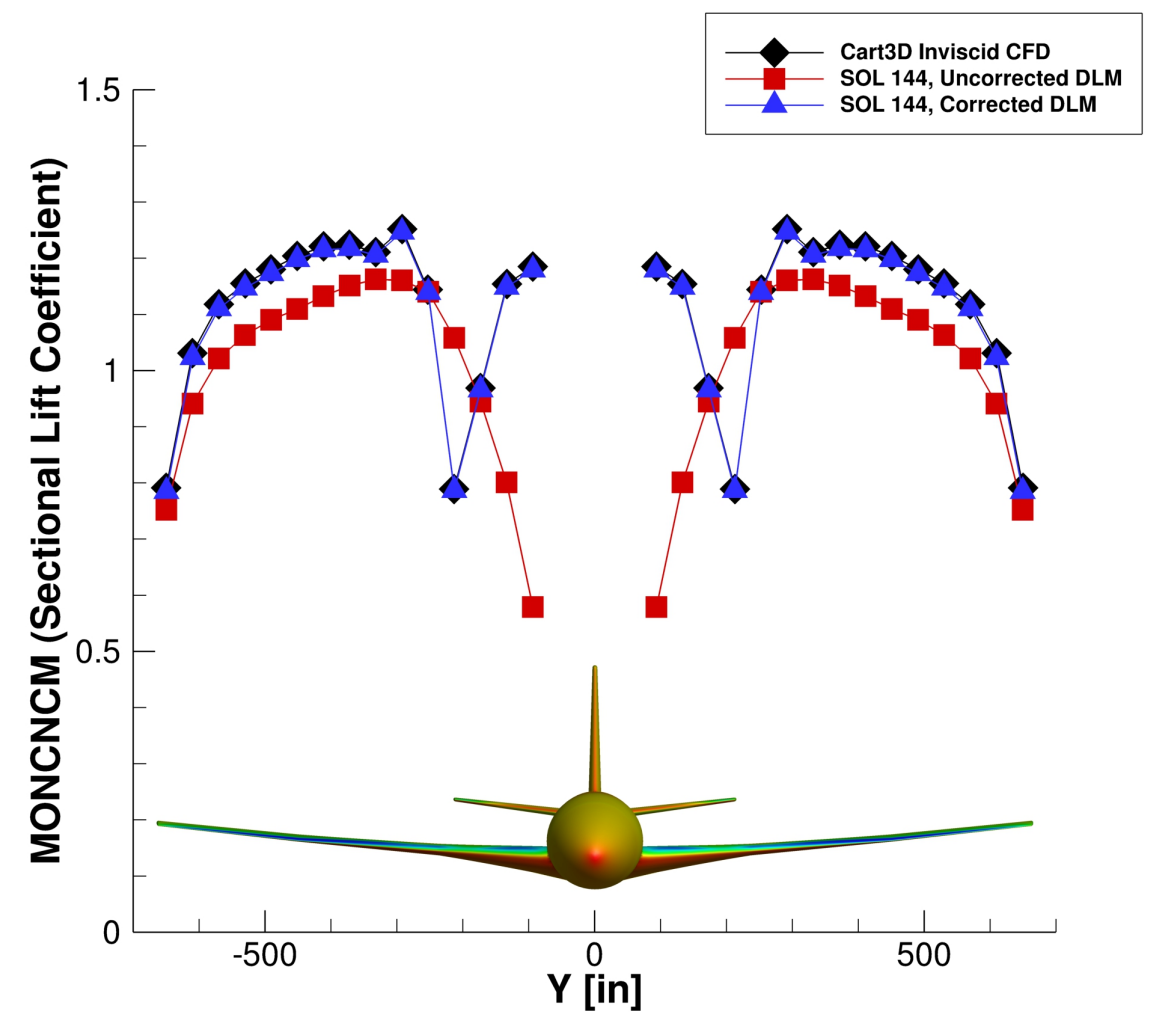

Figure 10. Comparison of sectional lift coefficient (MONCNCM Nastran output) for the main wing of the 737200 model for the: Cart3D CFD solution (black line/diamonds); uncorrected DLM solution (red line/squares); and aeroMatch-corrected DLM solution (blue line/triangles). A streamwise view of the 737-200, colored by contours of $C_{p}$, is shown at the bottom of the line plot for reference. 


\section{NASA Double Bubble Configuration}

Instigated in large part by the promising studies $^{3,4,18}$ led by researchers at the Massachusetts Institute of Technology (MIT), the Advanced Air Transport Technology (AATT) project's Systems Analysis and Integration (SA\&I) team is leading the conceptual design and analysis for a NASA aircraft featuring a double-bubble fuselage and boundary layer ingestion (BLI) technologies. Dubbed the NASA double-bubble concept, or ND8, this study seeks to use NASA state-of-the-art modeling and simulation techniques and tools to design a transport aircraft leveraging the fundamental technologies characterizing the original D8 concept.

Historically, FLOPS would have been used to estimate a weight buildup using high-level geometry and systems data; however, the empirical regression-based methods FLOPS utilizes rely on historical training data comprised of conventional, known, flying aircraft designs. Furthermore, FLOPS lacks the ability to actively consider physics-based design constraints, such as flutter, for weight estimation. In fact, one weakness of FLOPS is its tendency to increase wing span to unreasonable lengths as a way to increase aerodynamic performance. Often, however, this use leads to wing aspect ratios likely to violate flutter boundaries.

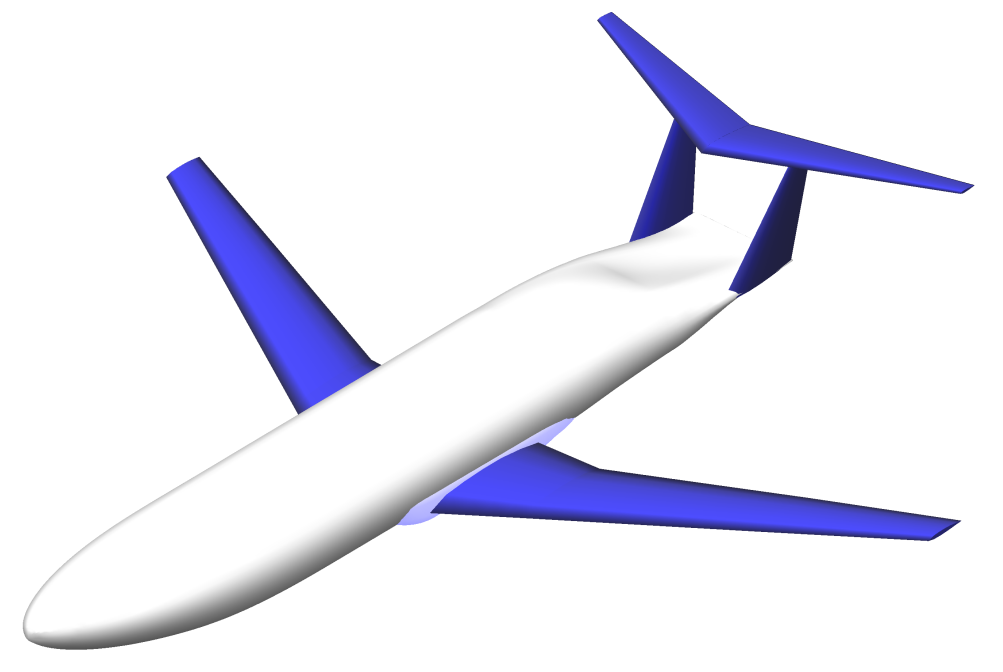

Figure 11. An OpenVSP rendering of the ND8 aircraft OML used for the present study.

The ND8 model, whose OML is shown in Fig. 11, is marked by its widened fuselage and integrated BLI engines at the rear of the fuselage (not shown here). Enabled by the double-bubble internal structure, the widened fuselage offers additional lift and additional interior cabin space over conventional tubular fuselages. Detailed analysis and systems-level trades on the fuselage structural design focused on concepts ranging from cusped, double-bubble structural architectures to smoothly contouring, oval structural architectures including centerline tension members and aerodynamic fairings, ${ }^{19}$ which are inline with the modeling approach taken by HCDstruct.

The current ND8 configuration is used in the present paper to demonstrate the modeling capabilities offered by HCDstruct 2.1.0. Details of the aerostructural FEM for the ND8 are presented below in Section IV.A. Next, in Section IV.B, the CFD analysis of the ND8 is presented in support of the subsequent application of aeroMatch, for which results are presented in Section IV.C.

\section{A. Aerostructural Model}

An aerostructural FEM of the ND8 aircraft concept shown in Fig. 11 was constructed using HCDstruct 2.1.0. This model consists of the primary structure FEM and a coupled DLM aerodynamics model. A rendering of the complete aerostructural FEM is presented in Fig. 12, where major aerodynamic model features are annotated. The FEM for the primary structure features a double-bubble fuselage topology constructed using CQUAD4 elements, with a marked cusp along the streamwise centerline and including an internal tensionbearing wall. The primary wing structure, including front and rear spars and skins comprising a wingbox, is connected to the fuselage structural model using Nastran's glued contact theory utilities. The horizontal and 
vertical tails are modeled using RBAR elements at the quarter chords, with concentrated mass (CONM2) elements for modeling their inertial loads. Two engine models comprised of RBAR and CONM2 elements are mounted at the rear fuselage bulkhead to model the inertial loadings of the engines. Finally, landing gear comprised of RBAR and CONM2 elements are placed at representative locations along the airframe.

The aerodynamic model of the ND8 includes DLM panel element (CAERO1) models for the main wing and tail lifting surfaces and a slender body element (CAERO2) model for the fuselage. While not annotated here, this aerodynamic model includes elevator, rudder, and aileron control surfaces, which are configured using AESURF cards. The aerodynamic forces arising from these aerodynamic model elements are splined to the primary structural elements, where the main wing forces are splined to the main wing spars, the tail forces are splined to the tail RBAR elements, and the fuselage forces are splined to the fuselage structure centerline.

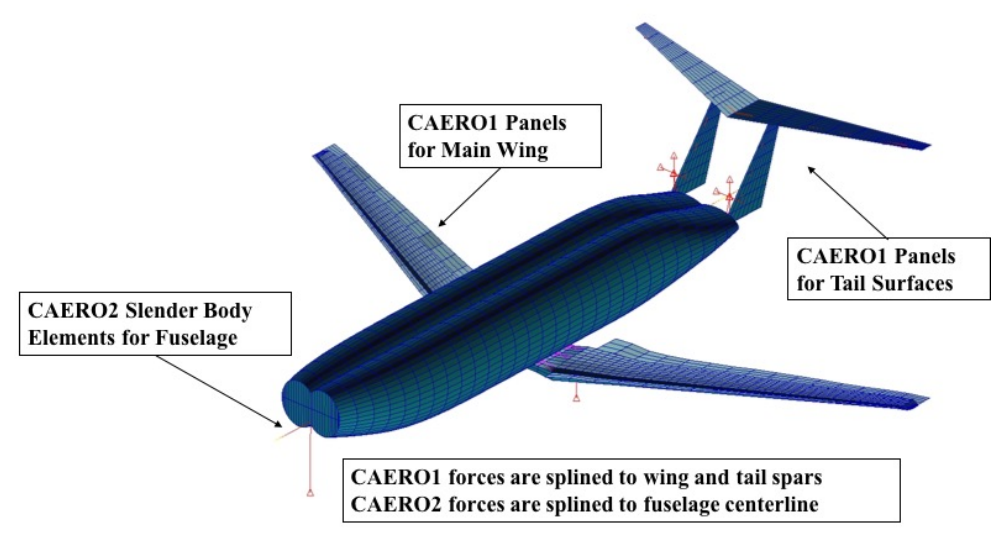

Figure 12. An annotated depiction of the ND8 aerostructural FEM, showing relevant aerodynamic model features.

The complete aerostructural model of the ND8 consists of nearly 4000 CQUAD4 elements, 706 CAERO1 panels, 148 CAERO2 elements, 60 RBAR elements, and 83 CONM2 masses. For the purposes of structural weight optimization, symmetry across the streamwise vertical centerplane is assumed, resulting in about 2000 PSHELL cards representing structural panel element thickness design variables for optimization using Nastran solution sequence 200. Details of the load cases used for optimization of the primary structure may be found in Ref. 2.

\section{B. CFD Analysis}

A similar CFD analysis to that performed for the 737-200 model described in Section III.B was repeated for the ND8, in which three-dimensional, inviscid, steady-state CFD simulations were performed using Cart3D in order to build a truth surface pressure solution with which aeroMatch could be used to formulate a $\left[W_{k k}\right]$ correction matrix. Solver configuration details for the ND8 were discussed in general terms in Section II.B; in this section, corresponding details of the computational mesh and solution are presented.

Like the 737-200 CFD analysis, three levels of mesh refinement were used to confirm mesh convergence, corresponding to 13, 14, and 15 levels of refinement beyond the initial Cartesian meshing supplied to Cart3D. The finest mesh solution, corresponding to 15 refinements, is used from this point forward. A depiction of the spanwise centerplane mesh is shown in Fig. 13, along with the surface mesh colored by contours of $C_{p}$. The computational mesh included approximately 129.2 million cells. The surface mesh was tailored to provide smooth transitions between aircraft components, such as at the wing and fuselage intersection, and to cluster cells around leading edges of the lifting surfaces, as shown in Fig. 14.

The ND8 was simulated at an AOA of $12.6^{\circ}$ to simulate a trimmed $2.5 \mathrm{G}$ maneuver using a structurallyoptimized, rigid aeroelastic model, derived in a similar fashion to that of the 737-200 described in Section III.A. The Cart3D solver was executed until the residual and integrated forces remained unchanged, requiring approximately 300 iterations at a CFL number of 1.0. The $C_{p}$ solution is shown in Fig. 15 for the entire vehicle. This $C_{p}$ surface solution was used by aeroMatch to build a corresponding $\left[W_{k k}\right]$ matrix, which is described in the next section.

\section{Results}

Whereas the application of aeroMatch to the 737-200 was done for validation purposes, the application of aeroMatch to the ND8 is shown here as the first application to an advanced aircraft concept and one for which the authors hypothesize the use of uncorrected DLM modeling is a particularly poor assumption due to the ND8's wide, lifting fuselage and relatively small wing planform. Similar to the 737-200 analysis, aeroMatch was used with the $C_{p}$ CFD solution shown in Fig. 15 to construct the DLM pre-multiplier matrix, 


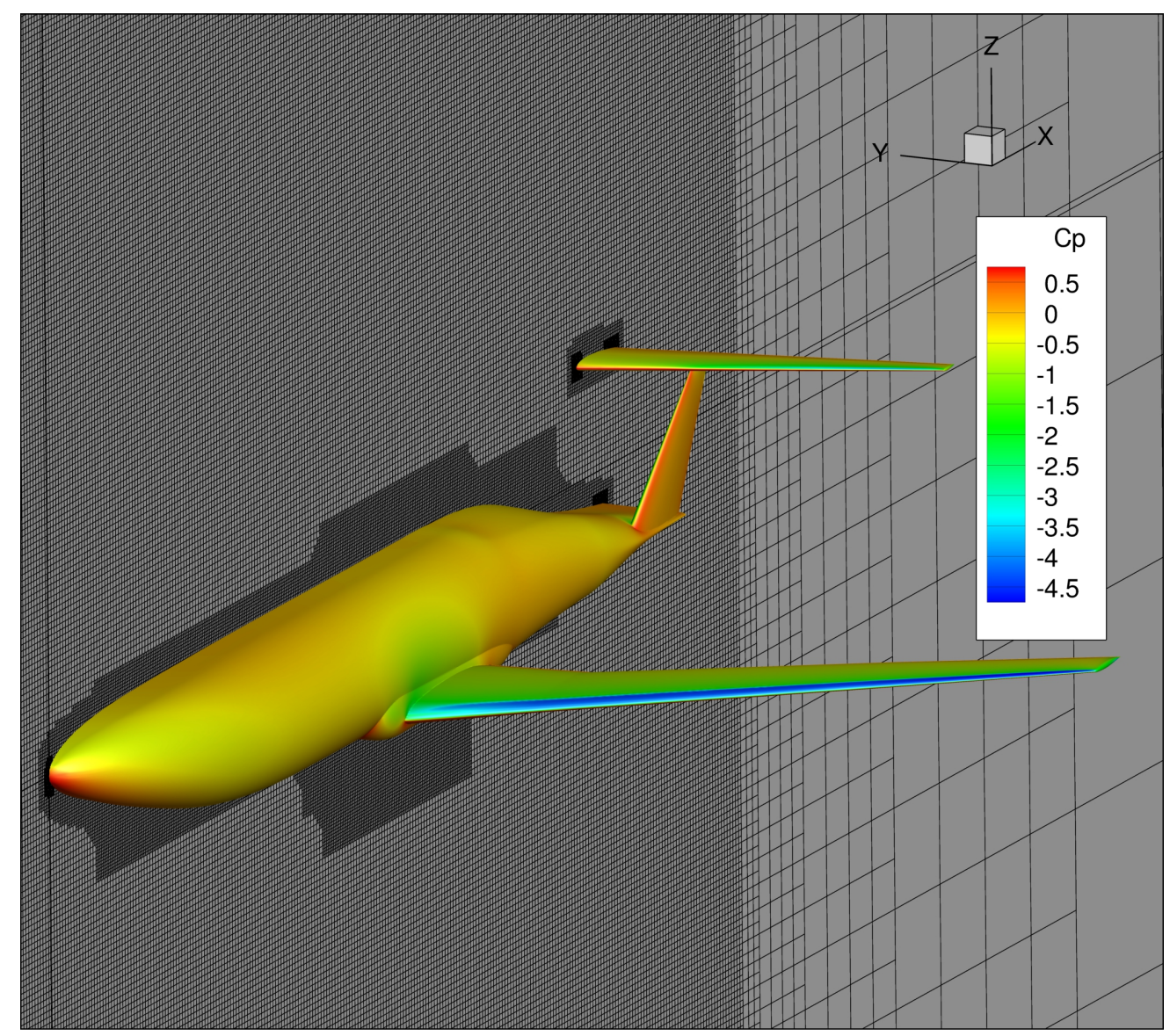

Figure 13. An illustration of the spanwise centerplane mesh away from the surface of the ND8, where the model surface is shown colored by contours of $C_{p}$.

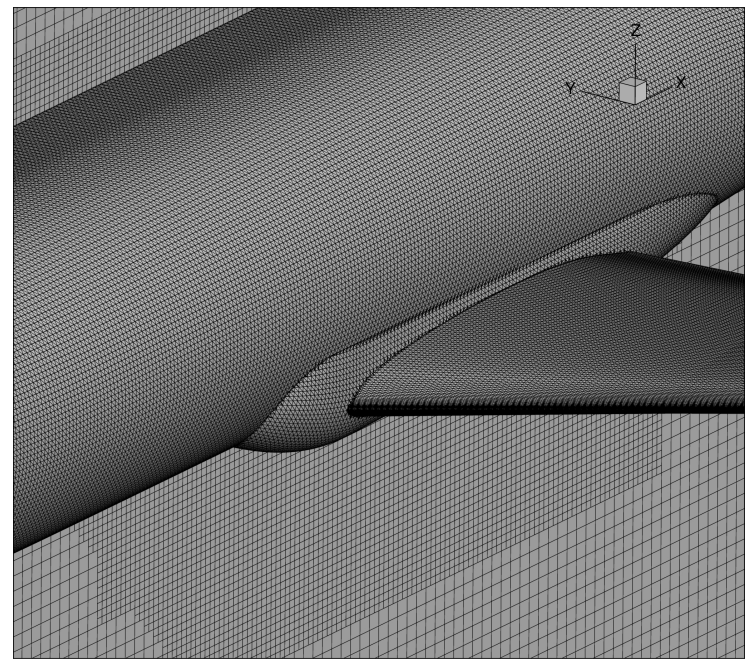

(a) Surface mesh detail at wing-fuselage intersection.

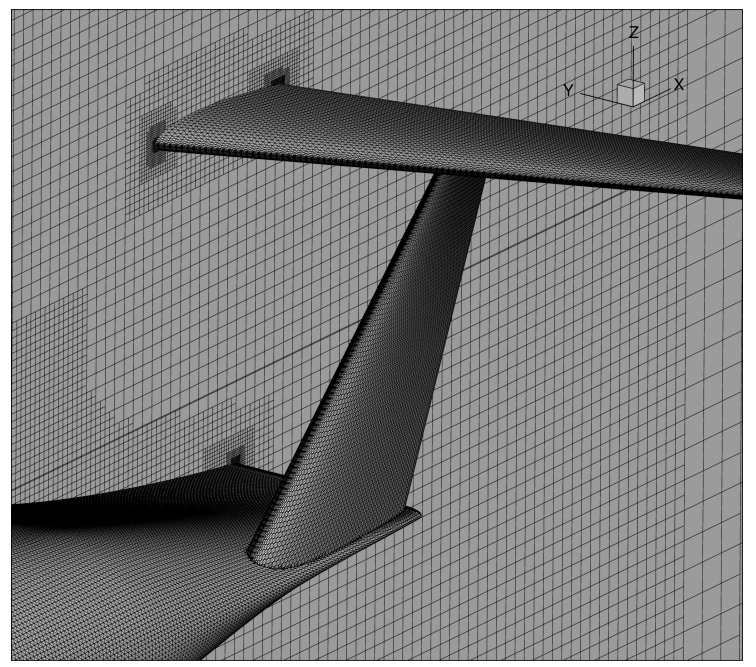

(b) Surface mesh detail at empennage.

Figure 14. Surface mesh illustrations showing the relative cell clustering at component intersections for the ND8 model. 


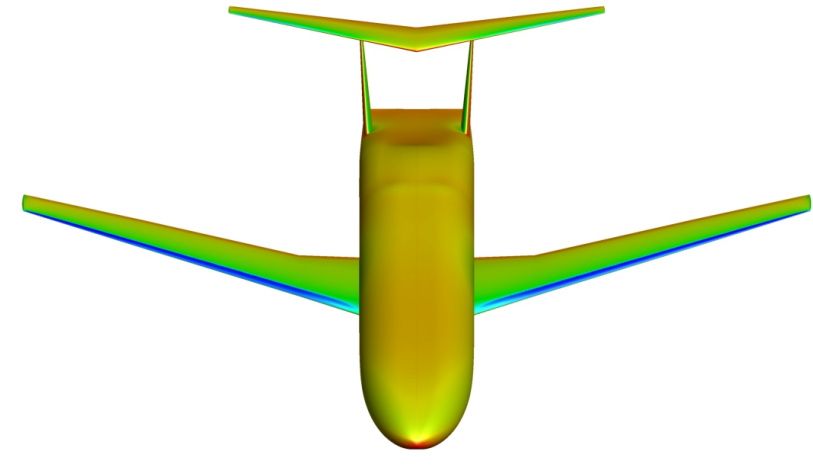

\begin{tabular}{|l|}
\hline Cp \\
0.5 \\
0 \\
-0.5 \\
-1 \\
-1.5 \\
-2 \\
-2.5 \\
-3 \\
-3.5 \\
-4 \\
\hline
\end{tabular}
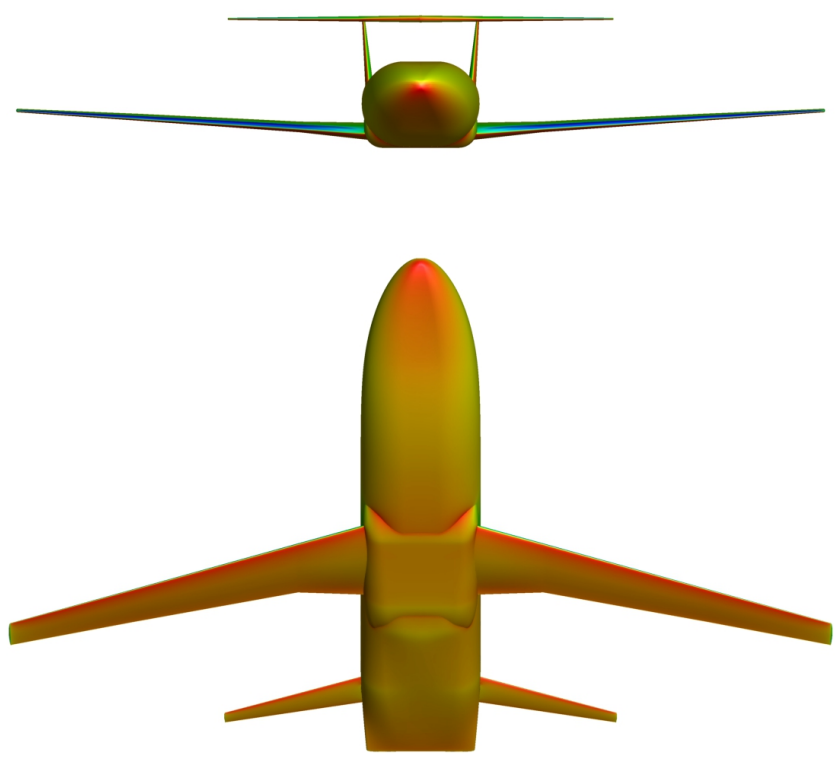

Figure 15. The ND8 $C_{p}$ surface solution for the fine mesh computed using Cart3D at an AOA of $12.6^{\circ}$. 
$\left[W_{k k}\right]$, shown in Fig. 16, for correcting the DLM aerodynamic predictions within SOL 144. Again, the ECFT correction was only applied to the main wing; thus, the resulting main wing sub-matrix of the $\left[W_{k k}\right]$ was augmented by an identity matrix such that the tail lifting surfaces and fuselage slender body elements were unaffected by the aerodynamic correction process, as can be seen by comparing the diagonal and off-diagonal elements of $\left[W_{k k}\right]$.

Comparisons of the sectional lift coefficient for the main wing for the uncorrected DLM model (red line/squares), the Cart3D CFD solution (black line/diamonds), and the aeroMatch-corrected DLM (blue line/triangles) are shown in Fig. 17. Also shown in this figure is a streamwise perspective of the ND8 model overlaid by contours of $C_{p}$ for referencing the spanwise data locations on the line plots of MONCNCM. The uncorrected DLM solution includes the effects of fuselage-wing interaction through the inclusion of the wing DLM panels in the fuselage interference group. Although the two outboard data stations show reasonable agreement between the uncorrected DLM and CFD solutions, elsewhere there is considerable disagreement. The uncorrected DLM data suggest considerably less sectional lift inboard of the wing tips and in particular near the fuselage. Inspecting the CFD solution data suggests the fuselage is producing considerable lift and that its interference effects are responsible for the increased sectional lift of the wing, relative to the uncorrected DLM solution. Given that the fuselage was designed to provide a substantial amount of total lift for this configuration, which has been shown for similar configurations using high-fidelity CFD simulations in previous studies, ${ }^{20,21}$ the substantial difference in generated lift at the inboard stations is unsurprising, as the DLM slender body elements are not suited for such applications. Further, comparing Fig. 17 to the previously reported results for the 737-200 model in Fig. 10, the significant drop in sectional lift at the midsection wing break for the 737-200 is not seen for the ND8. Although the ND8 model does have a midsection break, the relative change in sweep and taper ratio is considerably less than that of the 737-200. Additionally, the break is nearer to the wing root, and with the additional aerodynamic interference with the fuselage, it is unsurprising that the same decrease in section lift is not observed for the ND8. Generally, the disagreement between the CFD solution and the uncorrected DLM data further supports the notion that for advanced aircraft concepts, especially those that feature lifting fuselages, uncorrected DLM modeling inadequately captures the major aerodynamic features of the configuration.

Similar to the results shown for the 737-200 in Fig. 10, the corrected DLM sectional lift results agree well with the corresponding CFD data. Again, despite the considerable disagreement between the uncorrected DLM solution and the CFD data, the aeroMatch routine constructed a $\left[W_{k k}\right]$ matrix capable recovering the CFD section lift distribution well using the DLM modeling in SOL 144. This correction was applied only to the pitch mode of the aerodynamic decomposition matrix in the ECFT method, and future applications will leverage additional modes.

\section{Summary and Future Work}

Recent work on HCDstruct targeted the development and implementation of an aerodynamic-matching capability for increasing the accuracy of the aerodynamic modeling of advanced aircraft concepts for aeroelastic optimization studies. The methodology relies on the ECFT method, which uses a modal decomposition of the aerodynamic downwash matrix for the subsequent construction of a mode-selective pre-multiplier correction matrix. For the current implementation, construction of the correction matrix relies on a corresponding surface solution of $C_{p}$, which for the current work was the result of three-dimensional, full-configuration, inviscid CFD simulations. However, the aeroMatch utility is written such that the source of the correction $C_{p}$ solution is irrelevant. Specifically, the surface solution used for correction must be in an ASCII, default Tecplot format, but can be derived from any source, be it CFD, experiment, or otherwise.

The current study validated the current implementation of aeroMatch against a known, flying designthe Boeing 737-200 - and also illustrated its application to an advanced aircraft concept - the ND8. In both cases, the corrected DLM aerodynamics matched the corresponding CFD solution used to construct the correction matrix well. Further, in both cases, the results served to illustrate the need for such a correction capability, as the uncorrected DLM failed to predict prominent features of the sectional lift distributions computed using higher-fidelity CFD simulations.

With the current implementation completed and shown to correct Nastran's default DLM model, future work will include expanding this correction capability to include all vehicle lifting surfaces, versus being limited to the main wing, and to include the application of additional aerodynamic modes. Further, aeroMatch will be used to correct the aerodynamic modeling for static aeroelastic structural optimizations of the 


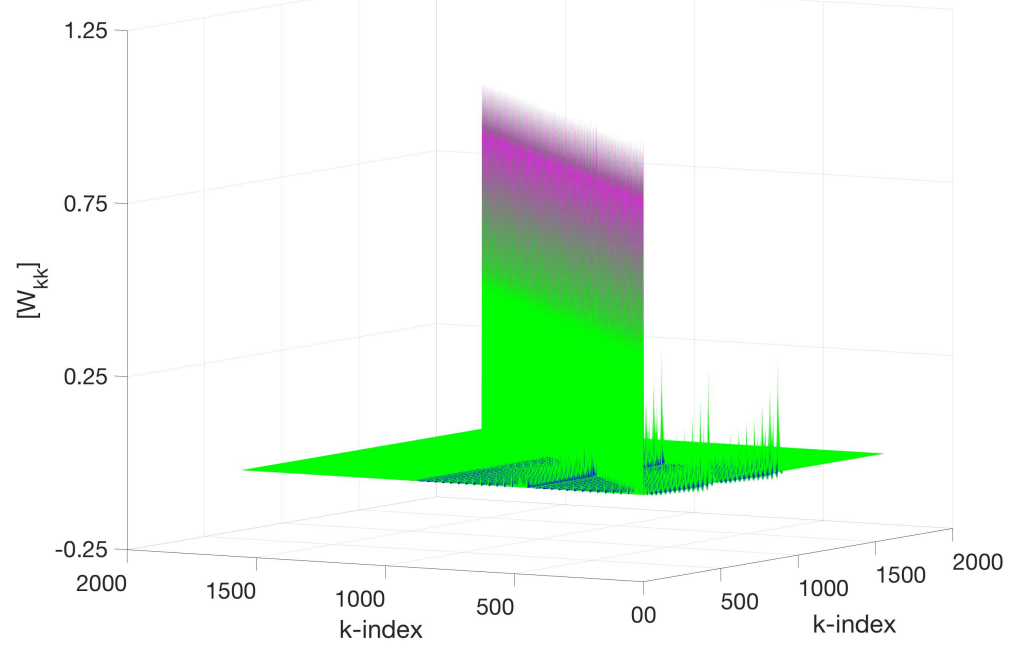

Figure 16. Surface plot of the $\left[W_{k k}\right]$ matrix as computed using aeroMatch for the ND8 model.

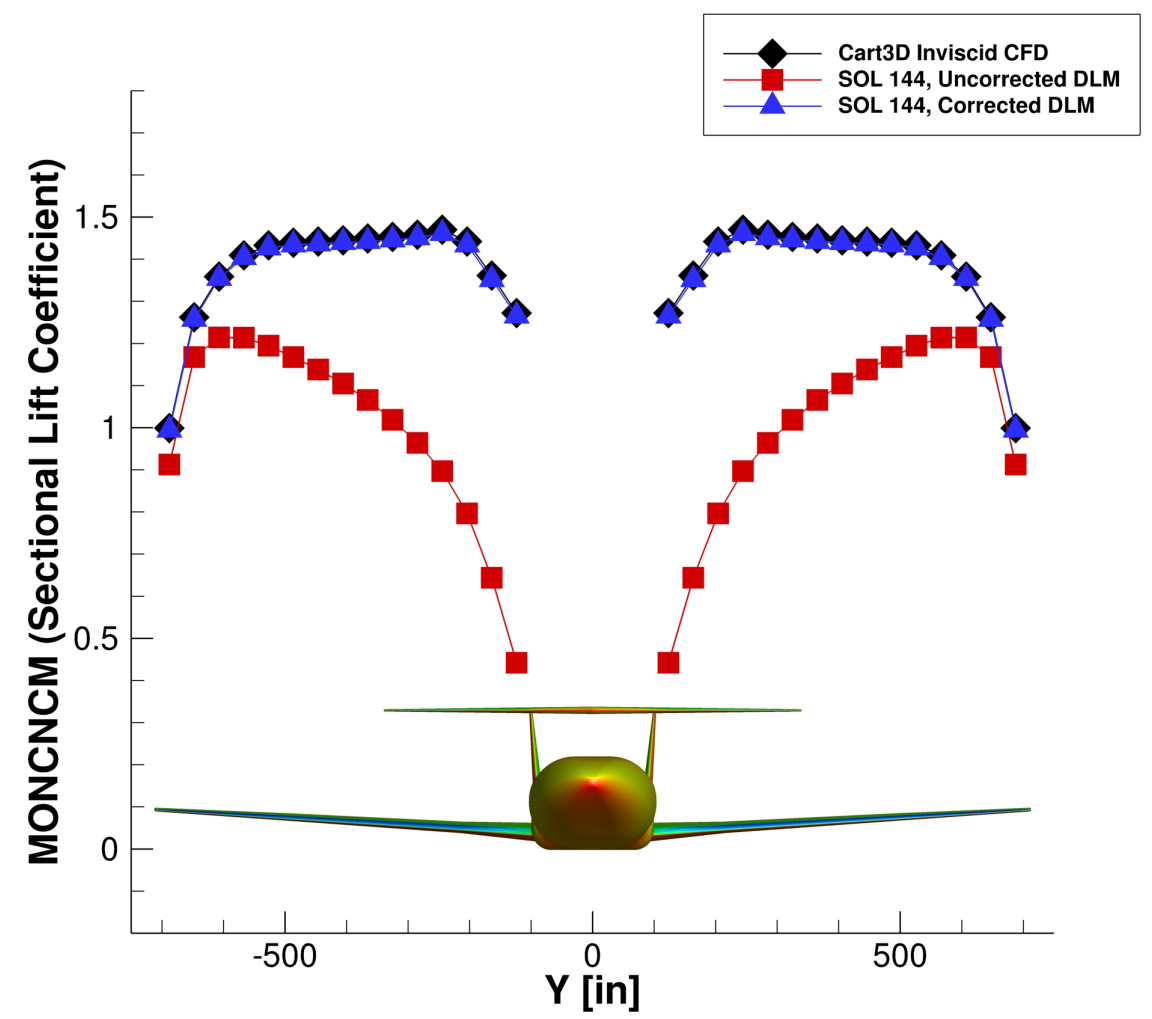

Figure 17. Comparison of sectional lift coefficient (MONCNCM Nastran output) for the main wing of the ND8 model for: Cart3D CFD solution (black line/diamonds); uncorrected DLM solution (red line/squares); and aeroMatch-corrected DLM solution (blue line/triangles). A streamwise view of the model, colored by contours of $C_{p}$, is shown at the bottom of the line plot for reference. 
737-200 and the ND8, before then being used to support flutter analysis of both models.

\section{Acknowledgments}

This work was supported by NASA's Advanced Air Transport Technology (AATT) and Transformational Tools and Technologies (TTT) projects. The authors thank Dr. Irian Ordaz for the numerous helpful discussions regarding the use of ModelCenter and Cart3D. Further, the authors thank Mr. Ty Marien and Dr. Jason Welstead for providing ND8 OpenVSP and FLOPS models.

\section{References}

${ }^{1}$ McCullers, L. A., FLOPS Flight Optimization System, FLOPS Users Manual, December 2009.

${ }^{2}$ Quinlan, J. R. and Gern, F. H., "Optimization of an Advanced Hybrid Wing Body Concept using HCDstruct Version 1.2," AIAA Aviation Conference, Washington, DC, June 2016.

${ }^{3}$ Drela, M., "Development of the D8 Transport Configuration," AIAA Applied Aerodynamics Conference, Honolulu, Hawaii, June 2011.

${ }^{4}$ Greitzer, E. M., Bonnefoy, P. A., De la Rosa Blanco, E., Dorbian, C. S., Drela, M., Hall, D. K., Hansman, R. J., Hileman, J. I., Liebeck, R. H., Lovegren, J., Mody, P., Pertuze, J. A., Sato, S., Spakovsky, Z. S., Tan, C. S., Hollman, J. S., Duda, J. E., Fitzgerald, N., Houghton, J., Kerrebrock, J. L., Kiwada, G. F., Kordonowy, D., Parrish, J. C., Tylko, J., Wen, E. A., and Lord, W. K., "N+3 Aircraft Concept Design and Trade Studies, Final Report," Tech. rep., NASA/CR-2010-216794/VOL1, NASA Langley Research Center, Hampton, VA, December 2010.

${ }^{5}$ Quinlan, J. R. and Gern, F. H., "Aeroelastic Optimization of Generalized Tube and Wing Aircraft Concepts using HCDstruct Version 2.0," AIAA SciTech Forum and Exposition, Dallas, TX, January 2017.

${ }^{6}$ Bradley, K. R., "A Sizing Methodology for the Conceptual Design of Blended-Wing-Body Transports," Tech. rep., NASA/CR-2004-213016, NASA Langley Research Center, Hampton, VA, September 2004.

${ }^{7} \mathrm{Li}, \mathrm{V}$. and Velicki, A., "Advanced PRSEUS Structural Concept Design and Optimization," 12th AIAA/ISSMO Multidisciplinary Analysis and Optimization Conference, Victoria, British Columbia, Canada, September 2008.

${ }^{8}$ Jadic, I., Hermann, T., Hartley, D., and Giri, J., "Generalized Aerodynamic Forces based on CFD and Correction Factor Techniques for AGARD Wing 445.6," AIAA/ASME/ASCE/AHS/ASC Structures, Structural Dynamics and Materials Exhibit, Seattle, WA, April 2001.

${ }^{9}$ Moreno, R., Fedime, K., Narisetti, R., and Taylor, P. F., "A Modification to the Enhanced Correction Factor Technique to Correlate with Experimental Data," AIAA/ASME/ASCE/AHS/ASC Structures, Structural Dynamics and Materials Exhibit, Kissimmee, FL 2015.

${ }^{10}$ Giesing, J. P., Kalman, T. P., and Rodden, W. P., "Correction Factor Techniques for Improving Aerodynamic Prediction Methods," Tech. rep., NASA/CR-144967, NASA Langley Research Center, Hampton, VA, May 1976.

${ }^{11}$ Jadic, I., Dayton, H., and Jagannath, G., "An Enhanced Correction Factor Technique for Aerodynamic Influence Coefficient Methods," MSC Aerospace User's Conference, 1999.

${ }^{12}$ MSC Nastran Aeroelastic Analysis User's Guide, MSC Software Corporation, Santa Ana, CA, 2004.

13 Tecplot 360 EX: User's Manual, Bellevue, WA, tecplot 360 ex 2017 release 1 ed., 2017.

${ }^{14}$ Aftosmis, M., "Cart3D Resource Website," https://www.nas.nasa.gov/publications/software/docs/cart3d/index.html [cited 15 April 2017].

${ }^{15}$ Hahn, A., "Vehicle Sketch Pad: A Parametric Geometry Modeler for Conceptual Aircraft Design," 48th AIAA Aerospace Sciences Meeting and Exhibit, Orlando, FL, January 2010.

${ }^{16}$ Ordaz, I. and Li, W., "Integration of Off-Track Sonic Boom Analysis in the Conceptual Design of Supersonic Aircraft," AIAA Aerospace Sciences Meeting, Orlando, FL, January 2011.

${ }^{17}$ Tinoco, E. N., Ball, D. N., and Rice, F. A., "PAN AIR Analysis of a Transport High-Lift Configuration," Journal of Aircraft, Vol. 24, No. 3, March 1987, pp. 181-187.

${ }^{18}$ Uranga, A., Drela, M., Greitzer, E. M., Titchener, N. A., Lieu, M. K., Siu, N. M., Huang, A. C., Gatlin, G. M., and Hannon, J. A., "Preliminary Experimental Assessment of the Boundary Layer Ingestion Benefit for the D8 Aircraft," AIAA Aerospace Sciences Meeting, National Harbor, MD, January 2014.

${ }^{19}$ Mukhopadhyay, V., Welstead, J. R., Quinlan, J. R., and Guynn, M. D., "Structural Configuration Systems Analysis for Advanced Aircraft Fuselage Concepts," AIAA Modeling and Simulation Technologies Conference, Washington, DC, June 2016.

${ }^{20}$ Pandya, S. A., "External AAerodynamic Simulations for the MIT D8 "Double-Bubble" Aircraft Design," Seventh International Conference on Compuational Fluid Dynamics, Big Island, HI, July 2012.

${ }^{21}$ Pandya, S. A., Huang, A., Espitia, A., and Uranga, A., "Computational Assessment of the Boundary Layer Ingesting Nacelle Design of the D8 Aircraft," AIAA SciTech, National Harbor, MD, January 2014. 